\title{
Characterisation of immune cells within the equine endometrium - a true challenge!
}

\author{
Nicole Rudolph, Heinz-Adolf Schoon and Sandra Schöniger \\ Institute of Pathology, Faculty of Veterinary Medicine, University of Leipzig, Leipzig, Germany
}

\begin{abstract}
Summary: In mares, endometrial diseases including endometritis are the main cause of pregnancy failure. A frequent finding in equine endometrial biopsies is non-suppurative endometritis. An immune mediated pathogenesis of this disease appears likely. A more detailed characterization of the involved lymphocyte and macrophage populations can be performed on zinc-salt fixed equine endometrial biopsies. The zinc-salt fixation is a cost-effective ready to use solution and permits the routine histologic diagnosis of endometrial diseases in mares. By using this method, the following immunohistochemical features of non-suppurative endometritis were revealed: It is a predominantly CD3 + T cell mediated disease with the frequent contribution of small numbers of CD20 + B cells and CD172a+ macrophages. Marked individual differences in the contribution of CD4 + helper T cells and CD8 + cytotoxic T cells exist, and the total number of CD3+ T cells often exceeded the sum of the CD4 + and CD8 + cells indicating the presence of CD4-/CD8- double negative T cells. Differences in the relative numbers of $\mathrm{CD} 3+, \mathrm{CD} 4+$ and $\mathrm{CD} 8+\mathrm{T}$ cells are also detected in endometria without inflammation. These results indicate that several forms of non-suppurative endometritis may exist and that individual factors likely contribute to differences in the immune cell composition of this disease.
\end{abstract}

Keywords: endometrium, mare, formalin and zinc-salt fixed tissue, immunohistochemistry, lymphocyte and macrophage subpopulations

Citation: Rudolph N., Schoon H-A., Schöniger S. (2017) Characterisation of immune cells within the equine endometrium - a true challenge! Pferdeheilkunde 34, 23-26; DOI 10.21836/PEM20180104

Correspondence: PD Dr. Sandra Schöniger, Institute of Pathology, Faculty of Veterinary Medicine, University of Leipzig, An den Tierkliniken 33,04103 Leipzig, Germany; sandra.schoeniger@vetmed.uni-leipzig.de

\section{Introduction}

Endometrial diseases including endometritis and degenerative disorders are the main cause of subfertility in mares (Schoon et al. 1997, Kenney and Doig 1986) and thus can have a major financial impact on the horse breeding industry (de Mestre 2013, LeBlanc and Causey 2009).

In equine endometrial biopsies the non-suppurative form of endometritis is mostly diagnosed (Ebert et al. 2014, Schoon et al. 1997). It is usually clinically inapparent and characterized by infiltration of the endometrium with lymphocytes, plasma cells and/or macrophages (Schoon et al. 1997, 1992). Thus, the microscopic examination of an endometrial biopsy is an important diagnostic tool for its diagnosis (Schoon et al. 1997). So far, the cause and pathogenesis of non-suppurative endometritis is unclear (Schoon et al. 1997) and therefore a specific treatment is not available (LeBlanc and Causey 2009). A participation of immune-mediated mechanisms is suspected.

The subclassification of the immune cell populations involved in non-suppurative endometritis will likely provide important information on the etiology and pathogenesis of this disease. In addition, it would likely be beneficial for the design of novel therapeutic options.

The more detailed characterization of the involved cell populations, however, imposes a major challenge. Commercially available antibodies for immunohistochemistry are frequently not specific for one particular immune cell subpopulation due to their partially overlapping receptor profiles (Taylor et al. 2005, Grünig et al. 1995) and are often established solely for immunohistochemistry on cryostat sections of native frozen equine tissue (Dakin et al. 2012, Flaminio et al. 2007, Watson and Thomson 1996). Regarding the former aspect the interpretation of the cellular immunostaining has to be performed under consideration of light microscopic findings. The cryostat method is impractical in a routine diagnostic setting, since endometrial biopsies have to be placed in fixative prior to their submission to prevent autolysis.

The characterization of the immune cell populations involved in equine non-suppurative endometritis requires the use of a fixation method that is cost-efficient and can be included in the routine diagnostic work-up of endometrial diseases and the prognostication of a mare's fertility.

\section{Material and Methods}

Fixation methods

Routinely, equine endometrial biopsies are fixed in 10\% neutral buffered formalin, since this fixation is most commonly used, because of its feasible use (Snider et al. 2011). Recently, the zinc-salt fixation has been revealed as an alternative fixation method for the routine diagnostic, since it allows comparable excellent tissue preservation as the formalin fixation (Huth et al. 2014, Beckstead 1994). Further, it was shown, that immunohistochemistry for the detection of CD4+ and CD8 + lymphocytes can be performed on zinc-salt fixed tissue as well, whereas the formalin fixation is unsuitable for the identification of several these cell populations in equine tissue (Huth et al. 2014). 
Moreover, immunohistochemical cell markers for the identification of macrophages were tested in comparison on formalin fixed and zinc-salt fixed tissue (Rudolph et al. 2017). Due to their limited reactivity in formalin fixed tissue, the further immunohistochemical characterization of lymphocytes and macrophage subpopulations as well as the histochemical identification of plasma cells was performed on zinc-salt fixed endometrial biopsies of mares with non-suppurative endometritis and without inflammation (Rudolph et al. 2017). Examined endometrial tissue samples were elected from a pool of zinc-salt fixed equine endometrial biopsies that had been submitted for routine microscopic examination and determination of fertility.

\section{Phenotyping of immune cells and their subpopulations}

This was performed by the immunohistochemical detection of the following antigens of lymphocyte and macrophage subpopulations: anti-CD3 (pan T lymphocyte marker), anti-CD4 (helper T cell marker), anti-CD8 (cytotoxic T cell marker), anti-CD20 (B lymphocyte marker), anti-CD172a (pan macrophage marker), anti-CD14 (M1 macrophage marker) and anti-CD206 (M2 macrophage marker). Plasma cells were identified by the use of the methyl green-pyronin stain. For this, serial sections of equine endometrial tissue samples were used (Rudolph et al. 2017, Huth et al. 2014).

Semiquantitative evaluation of immune cell numbers: Within each of the serial sections, the numbers of immunopositive cells as well as plasma cells were counted in five randomly selected high power fields within the stratum compactum. Since macrophages may express CD4 and CD8 antigens and neutrophils may stain CD172a and CD14 immunopositive, the immunohistochemical evaluation was performed under consideration of cell type specific morphological features and the histopathological findings to rule out false positive counts of particular cell populations (Rudolph et al. 2017, Huth et al. 2014).

\section{Results}

\section{Fixation methods}

The results of the routine stain (haemalaun and eosin stain), the applied histochemical stain for the identification of plas- ma cells (methyl green-pyronin stain) as well as the immunohistochemical detection of the investigated cellular markers are provided in the table 1. It was shown that the zinc-salt fixation provides excellent staining results for the histopathological evaluation as well as the immunohistochemical detection of the applied cellular markers (Rudolph et al. 2017, Huth et al. 2014).

\section{Phenotyping of immune cells and their subpopulations}

Phenotyping of immune cells was performed on endometrial biopsies of 24 mares with a mild non-suppurative endometritis and four endometria without inflammation. Histopathology of the former showed the restriction of the endometritis to the stratum compactum consistent with a superficial endometritis. The average cell count of $T$ lymphocytes within the stratum compactum was about $3 \times$ higher in endometria with endometritis than in those without inflammation (Rudolph et al. 2017).

Immunohistochemistry revealed that in all mares with endometritis the cellular infiltrates were mainly composed of CD3+ T lymphocytes, in 15 cases each the additional presence of a few CD20 + B lymphocytes and/or CD172a+ macrophages was detected. All inflamed endometria contained variable numbers of plasma cells (Rudolph et al. 2017).

The further phenotyping of CD3 + T lymphocytes detected the presence of marked individual differences in the percentage of CD4 + helper T cells and CD8 + cytotoxic T cells. In 22 mares, the total numbers of CD3 + lymphocytes exceeded moderately to markedly the sum of the counted CD4+ and CD8+ lymphocytes suggestive of the additional presence of CD4-/CD8double negative $\mathrm{T}$ lymphocytes. Further subclassification of macrophages identified few to low numbers of CD206+ macrophages within endometria of two mares; CD14+ macrophages were not detected (Rudolph et al. 2017).

All biopsies without inflammation contained a few CD3 + T lymphocytes within the stratum compactum, whereas CD20+ $B$ cells were not detected, occasional CD172a+ macrophages were present in one case and a few plasma cells in two mares. Similar as observed within inflamed endometria, the relation of CD4 + and CD8+ lymphocytes differed mar-

\begin{tabular}{|c|c|c|}
\hline Stains/Marker & Formalin Fixation & IHC Zinc Fixative ${ }^{1}$ \\
\hline Hemalaun and eosin stain & Excellent quality & Excellent quality \\
\hline Methyl green-pyronin stain & Excellent quality & Excellent quality \\
\hline CD3 & +++ & +++ \\
\hline CD4 & - & +++ \\
\hline CD8 & - & +++ \\
\hline CD20 & +++ & +++ \\
\hline CD172a & $+(+)$ & +++ \\
\hline CD14 & - & $+(+)$ \\
\hline CD206 & +++ & $+(+)$ \\
\hline
\end{tabular}

-, lack of immunostaining; $+(+)$, mild to moderate immunostaining; +++ , strong immunostaining

I IHC Zinc Fixative, BD Biosciences Pharmingen, Heidelberg, Germany 
kedly between individual mares. A few CD206+ macrophages occurred in one mare; CD14+ macrophages were not detected (Rudolph et al. 2017).

\section{Discussion}

The zinc-salt fixation is an excellent method to combine the routine histopathological examination of endometrial biopsies with the immunohistochemical evaluation of immune cells and their subpopulations. In contrast to the formalin fixation, it is suitable for the immunohistochemical phenotyping of all examined immune cell populations (Rudolph et al. 2017, Huth et al. 2014).

The occasional immune cells that were detected within endometria of mares without endometritis likely represented components of the uterine mucosal immune defense. Notably, differences in the relative numbers of $\mathrm{CD} 3+, \mathrm{CD} 4+$ and CD8 + lymphocytes existed between endometria of mares with and without endometritis. These findings suggest that non-suppurative endometritis may develop subsequent to an exaggerated immune stimulation of resident endometrial immune cell populations that can differ between individual mares. An alternative explanation would be the existence of different forms of non-suppurative endometritis, that areevoked by different stimuli and are associated with a characteristic mucosal immune response pattern, for example a CD4+ lymphocyte mediated helper $\mathrm{T}$ cell response, a CD8+ lymphocyte evoked cytotoxic immune reaction or a predominance of CD4-/CD8- double negative lymphocytes (Rudolph et al. 2017, Huth et al. 2014).

Future studies on a larger number of mares without inflammation and with non-suppurative endometritis are necessary for the further investigation of these hypotheses. Those studies may even help to find out, why some mares have an increased susceptibility to develop a persistent endometritis ("susceptible mares"). Results of these investigations will likely assist to reveal possible etiologies and predisposing conditions for non-suppurative endometritis and will likely provide further insights into its pathogenesis. This information will subsequently help to identify specific therapies or prophylactic regimes with the benefit to improve the genital health of the individual mare and to reduce financial losses of the horse breeding industry.

\section{Conclusion}

The zinc-salt fixation based on immunohistochemical phenotyping of immune cells can be easily incoorperated into the routine diagnostic work-up of endometrial biopsies, since this fixative is commercially available as ready to use solution and represents a cost efficient fixation method. Thus, it represents an important diagnostic tool for the identification of a mare $s$ endometrial immune response.

\section{References}

Beckstead J. H. (1994) A simple technique for preservation of fixation-sensitive antigens in paraffin-embedded tissues. J Histochem Cytochem. 42, 1127-1134; DOI 10.1177/42.8.8027531

Dakin S. G., Werling D., Hibbert A., Abayasekara D. R., Young N. J., Smith R. K., Dudhia J. (2012) Macrophage sub-populations and the lipoxin A4 receptor implicate active inflammation during equine tendon repair. PloS One. 7, e32333; DOI 10.1371/journal. pone.0032333

De Mestre A. (2013) Reproductive failure in horses: identifying the problem. Vet. Rec. 172, 42-43; DOI 10.1136/vr.f36

Ebert A., Schoon D., Schoon H. -A. (2014) Age related endometrial alterations in mares - biopsy findings of the last 20 years. Leipziger Blave Hefte: Proc. 8th International Conference on Equine Reproductive Medicine. 2, 230-232

Flaminio M. J., Ibrahim S., Lunn D. P., Stark R., Steinbach F. (2007) Further analysis of anti-human leukocyte mAbs with reactivity to equine leukocytes by two-colour flow cytometry and immunohistochemistry. Vet. Immunol. Immunopathol. 119, 92-99; DOI 10.1016/i.vetimm.2007.06.035

Grünig G., Triplett L., Canady L. K., Allen W. R., Antczak D. F. (1995) The maternal leucocyte response to the endometrial cups in horses is correlated with the developmental stages of the invasive trophoblast cells. Placenta. 16, 539-559

Huth N., Schöniger S., Sieme H., Bartmann C.-P., Schoon H.-A. (2014) Lymphocyte subpopulations in the equine endometrium: a methodical approach with diagnostic implications. Pferdeheilkunde. 30, 4-14; DOI 10.21836/PEM20140101

Kenney R. M., Doig P. A. (1986) Equine endometrial biopsy. In: Morrow D. A., editor: Current therapy in Theriogenology. 2nd ed. Philadelphia: WB Saunders, 723-729

LeBlanc M. M., Causey R. C. (2009) Clinical and subclinical endometritis in the mare: both threats to fertility. Reprod. Dom. Anim. 44 (Suppl. 3), 10-22; DOI 10.1111/i.1439-0531.2009.014 85.x

Rudolph N., Schoon H.-A., Schöniger S. (2017) Immunohistochemical characterisation of immune cells in fixed equine endometrial tissue: a diagostic relevant method. Pferdeheilkunde. 33, 524537; DOI 10.21836/PEM20170601

Schoon H.-A., Schoon D., Klug E. (1997) [The endometrial biopsy in the mare with regard to clinical correlations.] [in German]. Pferdeheilkunde. 13, 453-464; DOI 10.21836/PEM19970506

Schoon H.-A., Schoon D., Klug E. (1992) [Endometrial biopsies as an ancillary aid in diagnosis and prognosis of subfertility in the mare.] [in German]. Pferdeheilkunde. 8, 355-362; DOI 10.21836/PEM19920603

Snider T. A., Sepoy C., Holyoak G. R. (2011) Equine endometrial biopsy reviewed: Observation, interpretation, and application of histopathologic data. Theriogenology. 75, 1567-1581; DOI 10.1016/i.theriogenology.2010.12.013

Taylor P. R., Martinez-Pomares L., Stacey M., Lin H.-H., Brown G. D., Gordon S. (2005) Macrophage receptors and immune recognition. Annu. Rev. Immunol. 23, 901-944; DOI 10.1146/annurev. immunol.23.021704.115816

Watson E. D., Thomson S. R. (1996) Lymphocyte subsets in the endometrium of genitally normal mares and mares susceptible to endometritis. Equine Vet. J. 28, 106-110 